\title{
The identification of gene signature and critical pathway associated with childhood-onset type 2 diabetes
}

\author{
Keren Jia ${ }^{1}$, Yingcheng Wu ${ }^{1}$, Jingyi Ju ${ }^{1}$, Liyang Wang ${ }^{1}$, Lili Shi ${ }^{2}$, Huiqun Wu ${ }^{\text {Corresp., }}{ }^{2}$, Kui Jiang ${ }^{2}$, \\ Jiancheng Dong ${ }^{1}$ \\ 1 Medical School of Nantong University, Nantong, Jiangsu, China \\ 2 Department of Medical Informatics, Medical School of Nantong University, Nantong, Jiangsu, China \\ Corresponding Author: Huiqun Wu \\ Email address: wuhuiqun@ntu.edu.cn
}

In general, type 2 diabetes (T2D) usually occurs in middle-aged and elderly people. However, the incidence of childhood-onset T2D has increased all across the globe. Therefore, it is very important to determine the molecular and genetic mechanisms of childhood-onset T2D. In this study, the dataset GSE9006 was downloaded from the GEO (Gene Expression Omnibus database); it includes 24 healthy children, 43 children with newly diagnosed Type 1 diabetes (T1D), and 12 children with newly diagnosed T2D. These data were used for differentially expressed genes (DGEs) analysis and weighted coexpression network analysis (WGCNA). We identified 192 up-regulated genes and 329 down-regulated genes by performing DEGs analysis. By performing WGGNA, we found that blue module (539 genes) was highly correlated to cyan module (97 genes). Gene ontology (GO) and pathway enrichment analyses were performed to figure out the functions and related pathways of genes, which were identified in the results of DEGs and WGCNA. Genes with conspicuous logFC and in the high correlated modules were input into GeneMANIA, which is a plugin of Cytoscape application. Thus, we constructed the proteinprotein interaction (PPI) network (92 nodes and 254 pairs). Eventually, we analyzed the transcription factors and references related to genes with conspicuous logFC or highdegree genes, which were present in both the modules of WGCNA and PPI network. Current research shows that EGR1 and NAMPT can be used as marker genes for childhoodonset T2D. Gestational diabetes and chronic inflammation are risk factors that lead to the development of childhood-onset T2D. 
1 The identification of gene signature and critical pathway associated with childhood-onset type 2

2

3 Keren Jia $^{1}$, Yingcheng $\mathrm{Wu}^{1}$, Jingyi Ju${ }^{1}$, Liyang Wang ${ }^{1}$, Lili Shi ${ }^{2}$, Huiqun $\mathrm{Wu}^{* 2}$, Kui Jiang ${ }^{2}$,

4 Jiancheng Dong ${ }^{2}$

$5{ }^{1}$ Medical School of Nantong University, Nantong, 226001, China.

6 2Department of Medical Informatics, Medical School of Nantong University, Nantong, 226001,

7 China.

8 *Corresponding author: Huiqun $\mathrm{Wu}, \mathrm{MD}, \mathrm{PhD}$

9 Department of Medical Informatics, Medical School of Nantong University, Nantong, 226001, 10 China.

11 Email: wuhuiqun@ntu.edu.cn

12 Abstract

In general, type 2 diabetes (T2D) usually occurs in middle-aged and elderly people. However, the incidence of childhood-onset T2D has increased all across the globe. Therefore, it is very important to determine the molecular and genetic mechanisms of childhood-onset T2D. In this study, the dataset GSE9006 was downloaded from the GEO (Gene Expression Omnibus database); it includes 24 healthy children, 43 children with newly diagnosed Type 1 diabetes (T1D), and 12 children with newly diagnosed T2D. These data were used for differentially expressed genes (DGEs) analysis and weighted co-expression network analysis (WGCNA). We identified 192 up-regulated genes and 329 down-regulated genes by performing DEGs analysis. By performing WGGNA, we found that blue module (539 genes) was highly correlated to cyan module (97 genes). Gene ontology (GO) and pathway enrichment analyses were performed to figure out the functions and related pathways of genes, which were identified in the results of DEGs and WGCNA. Genes with conspicuous $\operatorname{logFC}$ and in the high correlated modules were input into GeneMANIA, which is a plugin of Cytoscape application. Thus, we constructed the protein-protein interaction (PPI) network (92 nodes and 254 pairs). Eventually, we analyzed the transcription factors and references related to genes with conspicuous logFC or high-degree 
genes, which were present in both the modules of WGCNA and PPI network. Current research shows that EGR1 and NAMPT can be used as marker genes for childhood-onset T2D. Gestational diabetes and chronic inflammation are risk factors that lead to the development of childhood-onset T2D.

\section{Introduction}

Diabetes mellitus is a chronically progressive, metabolic disease that affects multiple organs over a period of time. In a diabetic patient, blood glucose levels are high as the body either fails to produce insulin or cells are not sensitive enough to insulin. To ensure normal blood sugar levels, most diabetic patients have to take lifelong medications that affect the quality of life. Julie $\mathrm{R}$ et al. reported that the incidence of diabetes has increased phenomenally all over the world (Ingelfinger \& Jarcho 2017). It is really scary to know that the prevalence of diabetes had increased by $30.6 \%$ from 2005 to 2015 (Charlson et al. 2016). The onset of diabetes is associated with many factors, including other chronic diseases (Wang et al. 2017), social conditions (Whitworth et al. 2017), and even global warming (Blauw et al. 2017). In summary, diabetes is a chronic disease of complex etiology and poses a threat to global health.

Based on pathogenesis, diabetes can be mainly classified into three types: gestational diabetes, type 1 diabetes (T1D), and type 2 diabetes (T2D). In general, T1D is known as insulindependent diabetes and often occurs in children and adolescents. In patients with T1D, beta cells of islets undergo cell-mediated autoimmune destruction. As a result, the pancreas of T1D patients cannot synthesize and secrete insulin on its own. Furthermore, T2D is known as noninsulin-dependent diabetes mellitus, which is the most common type of diabetes. Approximately $90 \%$ of diabetic patients are diagnosed with T2D. It often occurs in patients above the age of 35 . In these patients, insulin secretion decreases either due to the lack of insulin receptors or impaired insulin receptors. Gestational diabetes is defined as a condition in which a normal woman develops diabetes during pregnancy. Gestational diabetes usually occurs in the middle or late stages of pregnancy. Some studies monitored women with gestational diabetes for six 
months after delivery. They found that 38 to $100 \%$ of these lactating mothers developed T2DM within six months of delivery (Kim et al. 2002). This suggests that there is an intrinsic link between gestational diabetes and T2D. In addition to these three types, there are some rare types of diabetes, including maturity onset diabetes of the young(MODY), maternally inherited diabetes and steroid diabetes. MODY and maternally inherited diabetes are primary and hereditary diseases. The genetic patterns of them are autosomal dominant and maternal inheritance, respectively. Steroid diabetes is a secondary metabolic disorder caused by excessive glucocorticoids in the body.

With the development of precision medicine, researchers can now pay more attention to patients with childhood-onset T2D. Wu et al(Wu et al. 2017) noted that although T2D is common in people above the age of 35, the incidence of T2D has continued to rise in children. Both children and adolescents have physiological conditions that are different from those of adults; therefore, researchers must focus on the prevention, clinical diagnosis, and drug treatment of childhood-onset T2D. Researchers in Mexico have conducted several cross-sectional studies on children and adolescents in the area. The results indicate that T2D and metabolic syndromerelated traits were highly inherited in Mexican children and adolescents (Mirandalora et al. 2017). Joyce et al (Joyce et al. 2017) found that statins increased the risk of childhood-onset T2D without causing dyslipidemia. Lee et al (Lee et al. 2018) found that the risk of developing T2D increased significantly in children and adolescents with mental disorders when they were exposed to atypical antipsychotics. Akhlaghi et al (Akhlaghi et al. 2016) analyzed whether the published anti-hyperglycemic drugs were safe and effective for children and adolescents. These studies have partially revealed some links of T2D in children and adolescents. Nevertheless, scientists still do not know the molecular mechanism of childhood-onset T2D. Therefore, it is necessary to identify the genes and pathways related to the pathogenesis of childhood-onset T2D. Then, we can develop special measures for the prevention and treatment of childhood-onset T2D. 
81 following three groups: healthy vs. T1D groups and the T1D vs. T2D groups. Unfortunately, childhood-onset T2D did not attract the attention of researchers. Hence, we still do not know the differences and similarities between healthy individuals and T2D patients. In this study, we identified the differentially expressed genes (DEGs) in the microarray data (GSE9006) of healthy group and T2D group. Then, we searched for genes closely related to T2D by performing weighted co-expression network analysis (WGCNA), which was further used to calculate the coexpression of genes. We performed functional enrichment analysis on DEGs and T2D genes. In addition, we constructed a protein-protein interaction (PPI) network for these genes to identify the ones that showed critical expression. Our research study focused on finding genes or pathways that are closely related to T2D and revealing the underlying molecular mechanisms.

\section{Materials and methods}

\section{Affymetrix microarray data}

Kaizer et al (Kaizer et al. 2007) measured and uploaded the dataset GSE9006 to gene expression omnibus (GEO) (http://www.ncbi.nlm.nih.gov/geo/). This dataset contained the gene expression of peripheral blood mononuclear cells (PBMCs), which were obtained from 24 healthy children, 43 children with newly diagnosed T1D, and 12 children with newly diagnosed T2D. The demographic information of volunteers was mentioned in Table 1. We selected data of healthy children and children with newly diagnosed T2D for further analysis. The dataset was based on the following platform: GPL97 [HG-U133B] Affymetrix Human Genome Array.

\section{DEG analysis}

In this study, we analyzed DEGs in healthy and T2D patients by using the limma package (Ritchie et al. 2015), which is the core component of Bioconductor and is widely used to process the microarray data. $|\log \mathrm{FC}| \geq 0.5$ and $\mathrm{P}<0.01$ was set as the cut-off criteria.

\section{WGCNA analysis}

To determine the co-expression module and the link between the module and the phenotype, 
106

107

108

109

110

111

112

113

114

115

116

117

118

119

120

121

122

123

124

125

126

127

128

129

130

we performed WGCNA of the normalized gene expression matrix by using WGCNA package (Langfelder \& Horvath 2008). It is important to note that WGCNA package is an effective data mining tool used to identify all kinds of modules that are highly related to a phenotype, including genes, miRNA, and LncRNA. In this study, the genes were divided into 21 modules, including 20 effective modules and 1 ineffective module (identified as gray module). We calculated the link between each module and co-expressed genes in healthy and T2D groups.

\section{GO and pathway enrichment analyses}

Gene ontology (GO) (Sherlock 2009) includes three types of data, namely, cellular components (CC), molecular functions $(\mathrm{MF})$, and biological processes (BP). GO is often used to annotate genes according to a defined set of structured words. The Kyoto Encyclopedia of Genes and Genomes (KEGG) (Kanehisa \& Goto 2000) is used to match the information of gene pathways. Reactome is a great tool to obtain gene-related pathways and interactions (Beloqui et al. 2009) . GO and pathway enrichment analyses are used to help us figure out the information of genes. In this study, GO and pathway enrichment analysis was performed on DEGs and the genes highly related to $\mathrm{T} 2 \mathrm{D}$. $\mathrm{P}<0.05$ was the criterion used for distinguishing non-meaningful pathways. At least two genes were required to enrich each pathway.

\section{PPI network analysis}

GeneMANIA software (Montojo et al. 2010) was used to establish gene interactions and to predict gene function. The PPI network was used to analyze DEGs and genes in both the highassociated modules. We set kappa $>0.4$ and $\mathrm{P}<0.05$ as the criteria. Network analysis was performed by running GeneMANIA software run in Cytoscape application. The critical hubs were the nodes that were highly connected to other nodes.

\section{Analysis of diseases and transcription factors related to critical genes}

The Comparative toxicogenomics database (CTD) (Davis et al. 2015) is often used to analyze the link between genes and diseases. In this study, we determined whether the target 
131 gene was linked to diabetes. In the CTD database, we searched all the genes that were reportedly 132 associated with T2D and T1D. Then, we matched these genes with our target genes. iRegulon 133 (Janky et al. 2014) is a plugin for Cystoscape application, which uses a set of pre-defined 134 transcription factors (TFs) and direct transcriptional targets to extract information related to TFs 135 in a group of co-expressing genes. Ultimately, the output is a set of transcription tracks and a list 136 of genes associated with the tracks. A network was constructed with the output results. To reflect 137 the reliability of results, the values of relevant parameters were set as follows: the maximum 138 false discovery rate on motif similarity $=0.001$; the minimum identity between orthologous genes $=0.05$, and the normalized enrichment score $(\mathrm{NES})=5$. Further analysis was performed on pairs for which $\mathrm{NES} \geq 5$.

141

142

143

144

145

146

147

148

149

150

151

152

153

154

155

156

\section{Results}

\section{$D E G$ analysis}

We compared the gene expression levels of healthy children and children with newly diagnosed T2D. Figure 1 showed that 521 genes had differential expression, including 192 upregulated genes and 329 down-regulated genes. The average $\operatorname{logFC}$ value of up-regulated genes was 0.787 , while the average $\log \mathrm{FC}$ value of down-regulated genes was -0.938 . The terms with $\log \mathrm{FC}>1.5$ or $\operatorname{logFC}<-1.5$ are EGR1 $(\operatorname{logFC}=2.005)$, HIST1H4PS1 $(\operatorname{logFC}=1.793)$, CELF4 $(\log \mathrm{FC}=1.665)$, CXorf56 $(\log \mathrm{FC}=1.573)$, PROK2 $(\log \mathrm{FC}=1.564)$, ORMDL3 $(\operatorname{logFC}=-$ 2.496), BF514098 (logFC = -1.846), MRPL39 ( $\operatorname{logFC}=-1.788)$, LINC00644 $(\operatorname{logFC}=-1.772)$, LOC90246 $(\log \mathrm{FC}=-1.770)$, POLB $(\operatorname{logFC}=-1.729)$, AA683356 (logFC $=-1.692)$, MIR497HG $(\log \mathrm{FC}=-1.653)$, AK026199 (logFC $=-1.616)$, AA885523 $(\log \mathrm{FC}=-1.592)$, VN1R3 $(\operatorname{logFC}=-1.588)$, TOX2 $(\operatorname{logFC}=-1.579)$, BF433815 $(\log \mathrm{FC}=-1.576)$, AW629036 $(\log \mathrm{FC}=-1.555), \mathrm{LRRC} 45(\operatorname{logFC}=-1.546), \mathrm{LRCH} 3(\log \mathrm{FC}=-1.533)$, TUG1 $(\operatorname{logFC}=-1.528)$, $\mathrm{BF} 110980(\operatorname{logFC}=-1.517), \operatorname{PLD} 5(\operatorname{logFC}=-1.513)$ and $\mathrm{CCDC} 102 \mathrm{~B}(\operatorname{logFC}=-1.504)$.

\section{WGCNA analysis}

Under the condition of soft threshold $=6$, each gene was divided into 21 modules (20 valid 
modules and 1 invalid module) by cluster analysis; the correlation of these genes with T2D phenotype was calculated. Figure 2 shows the correlation results. It can be seen that the blue and cyan modules have a significant correlation with T2D, which are $0.74(\mathrm{p}<0.01)$ and $0.69(\mathrm{p}<$ 0.01), respectively. The two modules contain a total of 636 genes; the blue module contains 539 genes, while the cyan module contains only 97 genes.

\section{GO and pathway enrichment analyses}

GO, Reactome, and KEGG pathway analyses were used to determine the up-regulated genes, the down-regulated genes, and the genes significantly correlated to T2D (blue and cyan). Table 2 displays the GO results, which indicated that the up-regulated genes were highly enriched in cytokine metabolic process, cytokine biosynthetic process, interleukin-1 beta production, positive regulation of cytokine biosynthetic process, and MyD88-dependent toll-like receptor(TLR) signaling pathway. The down-regulated expression of differential genes was mainly associated with the development of lymph vessel and fat pad. In T2D-related modules, the genes either conjugated or removed small proteins to perform functions, such as RNA binding, RNA processing, and protein modification. Some genes were further enriched in organelles' lumen, nuclear region, and other items.

Table 3 presents the results of KEGG and Reactome pathway analysis, indicating the involvement of up-regulated genes in TLR signaling pathway and in diseases of the immune system. In T2D-related modules, the genes were mainly involved in vasopressin-regulated water reabsorption pathways. The down-regulated expression of differential genes was mainly enriched through chromatin-modifying enzymes, chromatin organization, and mRNA splicing.

\section{PPI network analysis}

We performed PPI network analysis on 112 genes, which were present in both the DEGs and the highly correlated modules. As shown in Fig.3, we obtained 92 nodes and 254 pairs by setting the PPI score $>0.4$. An intersection was observed in genes of following types: the genes with $\log \mathrm{FC}>0.6$ or $\log \mathrm{FC}<-0.6$ in the DEGs, the genes with greater than average degree in the high 
183

184

185

186

187

188

189

190

191

192

193

194

195

196

197

198

199

200

201

202

203

204

205

206

207

208

correlation module, and the genes with greater than average degree in the PPI network. A total of 10 genes were obtained, all of which were up-regulated. The details were presented in Table 4. The 11 genes with the higher degree in the network are C14orf119 (logFC $=16)$, NAMPT $(\log \mathrm{FC}=15), \mathrm{NRBF} 2(\log \mathrm{FC}=14), \mathrm{MTO} 1(\log \mathrm{FC}=14)$, PIK3CG $(\operatorname{logFC}=13), \mathrm{RNF} 146$ $(\log \mathrm{FC}=13)$, VPS50 $(\operatorname{logFC}=13), \mathrm{CHM}(\log \mathrm{FC}=12), \mathrm{GPD} 2(\log \mathrm{FC}=12), \mathrm{RAB} 10(\operatorname{logFC}=$ $12)$ and ATAD1 $(\log \mathrm{FC}=12)$, which have the significant influence on the whole network.

Analysis of the transcription factors of maker genes

A total of 12 genes were used for further analysis. Among them, 10 important genes were obtained from PPI analysis. The remaining two genes were DEGs with large values of $\log \mathrm{FC}$, namely, EGR1 $(\operatorname{logFC}=2.00)$ and NAMPT $(\operatorname{logFC}=1.32)$. In the CTD database, these 12 genes are reportedly linked with T2D. In addition, we analyzed and compared their reports in T1D group. The results were shown in Table 5.

As shown in Fig.4, we analyzed the transcription factor regulatory network of these genes. We found that NES $\geq 5$ was for the following transcription factors: DEAF1 (NES = 7.114, degree = 6), GMEB2 (NES = 6.595, degree = 4), MAF1 (NES = 5.641, degree = 3), NKX3-2 $(\mathrm{NES}=5.287$, degree $=3), \mathrm{WDR} 83(\mathrm{NES}=5.041$, degree $=4)$.

\section{Discussion}

In humans, early growth response 1 (EGR1) is a protein-coding gene. Previous studies have reported that glucagon can transiently activate EGR1 in liver cells. To mediate glucagonregulated gluconeogenesis, hepatocytes up-regulate the expression of gluconeogenic genes. By blocking the function of EGR1 gene, we could increase glycogen content in hepatic cells, which would improve the tolerance toward pyruvate and lower fasting blood glucose. The gene EGR1 enhances insulin resistance in T2D patients with chronic hyperinsulinemia. Insulin resistance is one of the most significant causes of T2D (Shen et al. 2011). The gene EGR1 promotes the development of gestational diabetes by adversely impacting the glucagon-controlled gluconeogenesis (Zhao et al. 2016). In addition, EGR1 promotes also diabetic nephropathy, 
209

210

211

212

213

214

215

216

217

218

219

220

221

222

223

224

225

226

227

228

229

230

231

232

233

234

235

which is one of the most common complications of diabetes (Wang et al. 2015). Several findings have indicated that EGR1 promotes the inflammation within muscle and adipose (Fan et al. 2013) and also acts as an inflammatory mediator in reproductive tissues (Thakali et al. 2014). Indeed, the expression of EGR1 gene was unregulated in the placenta of obese women. A previous study has shown that the up-regulated expression of IL-8, IL-6, and TNF $\alpha$ was induced by EGR1 gene in BeWo cells (Saben et al. 2013). These findings indicated that EGR1 contributed to placental inflammation in obese women. Maternal adiposity must have triggered the expression of EGR1 in umbilical cord tissue (Thakali et al. 2014). Current research and previous studies have proved that EGR1 gene has significantly affected many aspects of diabetes and obesity. In multiple tissues of T2D patients, statistically significant differences were found in the expression of EGR1 gene.

Nicotinamide phosphoribosyltransferase (NAMPT) is a protein-coding gene in humans. Previous studies have reported that NAMPT is an adipocytokine that exhibits pro-inflammatory and immunoregulatory properties (Moschen et al. 2007) and regulates beta-cell insulin secretion (Revollo et al. 2007). Furthermore, NAMPT is involved in insulin resistance and chronic inflammation, which promotes the development of T2D (Ma et al. 2017) (Jaganathan et al. 2017) (Motawi et al. 2014). Obesity is an important factor that leads to the development of diabetes. In obese and overweight patients with metabolic syndrome, the expression of NAMPT increases in the plasma (Filippatos et al. 2007) and simulates the effect of insulin (Fukuhara et al. 2005). However, a previous study has reported that NAMPT is related to only T2D and not obesity (M et al. 2010). Some studies have reported plasma levels of visfatin, the product of NAMPT, increases in obese people (Jaleel et al. 2013), indicating that further research must be conducted to determine the relationship between NAMPT and obesity. Interestingly, the expression of NAMPT was found to be significantly higher in patients with gestational diabetes. This indicates that NAMPT is involved in the molecular mechanism of gestational diabetes (Krzyzanowska et al. 2006). Several studies have reported that Young women with gestational diabetes usually give birth to an overweight baby. This phenomenon has been attributed to intrauterine growth, which 
236

237

238

239

240

241

242

243

244

245

246

247

248

249

250

251

252

253

254

255

256

257

258

259

260

261

262

increases the prevalence of T2D in the offspring (Schaefergraf et al. 2005). Because NAMPT is also conspicuously up-regulated in children with T2D, it is necessary to further study the genetic mechanism of NAMPT in gestational diabetes patients and their offspring. These findings suggest that NAMPT is a marker gene in patients with childhood-onset T2D.

In this study, we also found that chronic inflammation was somewhat related to childhoodonset T2D. Thus, childhood-onset T2D might be caused by multiple risk factors. For the treatment and prevention of childhood-onset T2D, we need to correctly identify the contribution and sequence of risk factors. Hence, further studies must be conducted to confirm whether gestational diabetes and chronic inflammation are closely related to childhood-onset T2D.

According to KEGG, up-regulated genes are mainly enriched in the signaling pathways of TLR. The enrichment results of Reactome pathways indicated that up-regulated genes were related to the TLR Cascades, suggesting that TLRs play a pivotal role in childhood-onset T2D. Reactive oxygen is produced excessively in obese people, which leads to an imbalance in the endogenous antioxidant capacity and causes oxidative stress in adipocytes (Houstis et al. 2006). Obesity also promotes the excessive production of pro-inflammatory adipokines, which further aggravate the chronic inflammation of adipocytes (Ouchi et al. 2011). In fact, several studies have explained the association between obesity and chronic inflammation. These studies have further reported that chronic inflammation causes insulin resistance in obese people. Insulin resistance is a precursor to T2D in adults. These studies mainly investigated the adipose tissue of adults. In this study, we found that TLR signaling pathways mainly enriched up-regulated genes in PBMCs. In fact, TLR2 and TLR4 are important cell membrane receptors that elicit innate immune responses to infection (Tack et al. 2012) (Chmelar et al. 2013) (A et al. 2003). Previous studies have shown that TLR2 / 4 and JNK signaling pathways play a pivotal role in activating CD11c $(+)$ myeloid proinflammatory cells, which further promotes inflammation and subsequent insulin resistance in cells (Nguyen et al. 2007). The TLR4 signaling pathway participates in JNK activation and instigates palmitate-induced apoptosis of INS-1 $\beta$ cells. With the knockout of TLR4, we blocked palmitate-induced apoptosis of INS-1 cells; however, no such phenomenon 
was observed with the knockdown of TLR2 (Lee et al. 2011). The inflammatory factors produced during immunization play an important role in obesity-related T2D (Iiu et al. 2013). Some immune-related diseases are complications of diabetes. For example, some diabetic patients may develop a chronic airway inflammation, which further causes asthma. The above results indicate that children may develop chronic inflammation, which further induces insulin resistance and promotes the development of T2D. In addition, we also found that several other genes, such as PIK3CG, ZNF420 were also functionally related to inflammation and immune response (Hawkins \& Stephens 2007) (Tian et al. 2009). Therefore, this result further validates our findings.

In previous studies, animal models or elderly patients were investigated to elucidate the mechanism of T2D. Ours is the first study to partially elucidate the molecular mechanism of childhood-onset T2D with the help of bioinformatics. We found the marker genes (EGR1, NAMPT) and TLR signaling pathways of childhood-onset T2D. The up-regulated expression of EGR1 and NAMPT in PBMCs seems to be a gene marker of childhood-onset T2D. The results were compared with the gene expression of middle-aged and older patients with T2D. Thus, there is a genetic mechanism of NAMPT in gestational diabetes patients and their offspring. Such an offspring will have an increased risk of developing childhood-onset T2D. Children are less affected by environmental factors than adults, which allows us to speculate that genetic factors have more influence on childhood-onset T2D. Our findings suggest that NAMPT may be the key to understanding this issue. In addition, TLRs are important proteins involved in nonspecific immunity and are a bridge linking specific and non-specific immunity. TLR4 recognizes not only foreign pathogens, but also endogenous substances and degradants. Given the differences in the immune system between children and adults, as well as the special circumstances in which the fetus grows in the uterus, we believe that in children with T2D, the factors that activate the TLR signaling pathway cannot be equated with those of adults. The above results only analyze the underlying mechanisms of EGR1 and NAMPT in children with T2D. We present more details as well, including genes with higher $\log \mathrm{FC}$ and specific $\operatorname{logFC}$ 
values, genes with higher degrees and associated values, and information on transcription factors, which provide ideas for subsequent research on childhood-onset T2D. It must be emphasized that the physical condition of a child is very different from that of an adult. Therefore, future research studies must focus on elucidating the mechanisms of occurrence, prevention strategies, and treatment of children and adolescents with T2D.

\section{Conclusion}

EGR1 and NAMPT can be used as marker genes for childhood-onset T2D, and gestational diabetes and chronic inflammation are risk factors that lead to the development of childhoodonset T2D.

\section{References}

Andreas F, Anthony J G H, Russell P T, Ralph D and Steven M H. 2003. Inflammation in the prediabetic state is related to increased insulin resistance rather than decreased insulin secretion. Circulation 108:1822-1830.

Akhlaghi F, Matson KL, Mohammadpour AH, Kelly M, and Karimani A. 2016. Clinical Pharmacokinetics and Pharmacodynamics of Antihyperglycemic Medications in Children and Adolescents with Type 2 Diabetes Mellitus. Clinical Pharmacokinetics:1-11.

Beloqui A, Guazzaroni ME, Pazos F, Vieites JM, Godoy M, Golyshina OV, Chernikova TN, Waliczek A, Silva-Rocha R, and Al-Ramahi Y. 2009. Reactome array: forging a link between metabolome and genome. Science 326:252.

Blauw LL, Aziz NA, Tannemaat MR, Blauw CA, de Craen AJ, Pijl H, and Rensen PC. 2017. Diabetes incidence and glucose intolerance prevalence increase with higher outdoor temperature. Bmj Open Diabetes Research \& Care 5:e00317.

Charlson FJ, Erskine HE, Ferrari AJ, Leung J, Whiteford HA, Abajobir AA, Knibbs LD, Lalloo R, Scott JG, and Guo Y. 2016. Global, regional, and national incidence, prevalence, and years lived with disability for 310 diseases and injuries, 1990-2015: a systematic analysis for the Global Burden of Disease Study 2015.

Chmelar J, Chung KJ, and Chavakis T. 2013. The role of innate immune cells in obese adipose tissue inflammation and development of insulin resistance. Thrombosis \& Haemostasis 109:399-406.

Davis AP, Grondin CJ, Lennonhopkins K, Saracenirichards C, Sciaky D, King BL, Wiegers TC, and Mattingly CJ. 2015. The Comparative Toxicogenomics Database's 10th year anniversary: update 2015. Nucleic Acids Research 43:D914.

Fan YY, Ye GH, Lin KZ, Yu LS, Wu SZ, Dong MW, Han JG, Feng XP, and Li XB. 2013. Time-dependent expression and distribution of Egr-1 during skeletal muscle wound healing in rats. Journal of Molecular Histology 44:75-81. 
325

326

327

328

329

330

331

332

333

334

335

336

337

338

339

340

341

342

343

344

345

346

347

348

349

350

351

352

353

354

355

356

357

358

359

360

361

362

363

364

365

Filippatos TD, Derdemezis CS, Kiortsis DN, Tselepis AD, and Elisaf MS. 2007. Increased plasma levels of visfatin/pre-B cell colony-enhancing factor in obese and overweight patients with metabolic syndrome. Journal of Endocrinological Investigation 30:323-326.

Fukuhara A, Matsuda M, Nishizawa M, Segawa K, Tanaka M, Kishimoto K, Matsuki Y, Murakami M, Ichisaka T, and Murakami H. 2005. Visfatin: A Protein Secreted by Visceral Fat That Mimics the Effects of Insulin. Science 307:426-430.

Hawkins PT, and Stephens LR. 2007. PI3K $\gamma$ Is a Key Regulator of Inflammatory Responses and Cardiovascular Homeostasis. Science 318:64-66.

Houstis N, Rosen ED, and Lander ES. 2006. Reactive oxygen species have a causal role in multiple forms of insulin resistance. Nature 440:944.

Iiu S, Khkh S, Plotnikova OA, Alekseeva RI, Sentsova TB, and Vorozhko IV. 2013. [Markers of immune inflammation in patients with type 2 diabetes and obesity]. Voprosy Pitaniia 82:46-50.

Ingelfinger JR, and Jarcho JA. 2017. Increase in the Incidence of Diabetes and Its Implications. New England Journal of Medicine 376:1473.

Jaganathan R, Ravindran R, and Dhanasekaran S. 2017. Emerging Role of Adipocytokines in Type 2 Diabetes as Mediators of Insulin Resistance and Cardiovascular Disease. Canadian Journal of Diabetes.

Jaleel A, Aheed B, Jaleel S, Majeed R, Zuberi A, Khan S, Ahmed B, Shoukat F, and Hashim H. 2013. Association of adipokines with obesity in children and adolescents. Biomarkers in Medicine 7:731-735.

Janky R, Verfaillie A, Imrichová H, Van dSB, Standaert L, Christiaens V, Hulselmans G, Herten K, Naval SM, and Potier D. 2014. iRegulon: from a gene list to a gene regulatory network using large motif and track collections. Plos Computational Biology 10:e1003731.

Joyce N, Zachariah JP, Eaton CB, Trivedi AN, and Wellenius GA. 2017. Statin Use and the Risk of Type 2 Diabetes Mellitus in Children and Adolescents. Academic Pediatrics.

Kaizer EC, Glaser CL, Chaussabel D, Banchereau J, Pascual V, and White PC. 2007. Gene expression in peripheral blood mononuclear cells from children with diabetes. Journal of Clinical Endocrinology \& Metabolism 92:3705-3711.

Kanehisa M, and Goto S. 2000. KEGG: Kyoto Encyclopedia of Genes and Genomes. Nucleic Acids Research 27:29-34.

Kim C, Newton KM, and Knopp RH. 2002. Gestational Diabetes and the incidence of T2DM: A systematic review. Diabetes Care 25:1862-1868.

Krzyzanowska K, Krugluger W, Mittermayer F, Rahman R, Haider D, Shnawa N, and Schernthaner G. 2006. Increased visfatin concentrations in women with gestational diabetes mellitus. Clinical Science 110:605.

Langfelder P, and Horvath S. 2008. WGCNA: an R package for weighted correlation network analysis. Bmc Bioinformatics 9:559.

Lee H, Song DH, Kwon JW, Han E, Chang MJ, and Kang HY. 2018. Assessing the risk of type 2 diabetes mellitus among children and adolescents with psychiatric disorders treated with 
366

367

368

369

370

371

372

373

374

375

376

377

378

379

380

381

382

383

384

385

386

387

388

389

390

391

392

393

394

395

396

397

398

399

400

401

402

403

404

405

406

atypical antipsychotics: a population-based nested case-control study. European Child \& Adolescent Psychiatry:1-14.

Lee SM, Choi SE, Lee JH, Lee JJ, Jung IR, Lee SJ, Lee KW, and Kang Y. 2011. Involvement of the TLR4 (Toll-like receptor4) signaling pathway in palmitate-induced INS-1 beta cell death. Molecular \& Cellular Biochemistry 354:207-217.

Laudes M, Oberhauser F, Schulte DM, Freude S, Bilkovski R, Mauer J, Rappl G, Abken H, Hahn M, Schulz O and Krone W. 2010. Visfatin/PBEF/Nampt and resistin expressions in circulating blood monocytes are differentially related to obesity and type 2 diabetes in humans. Hormone \& Metabolic Research 42:268-273.

Ma X, An L, and Wang Q. 2017. Changes in Serum Nampt Levels and Its Significances in Diabetic Nephropathy Patients-The Potential Role of Nampt in T2DM with Diabetic Nephropathy. Endocrine Metabolic \& Immune Disorders Drug Targets 17:114.

Mirandalora AL, Vilchisgil J, Molinadíaz M, Floreshuerta S, and Klünderklünder M. 2017. Heritability, parental transmission and environment correlation of pediatric-onset type 2 diabetes mellitus and metabolic syndrome-related traits. Diabetes Res Clin Pract:151-159. Montojo J, Zuberi K, Rodriguez H, Kazi F, Wright G, Donaldson SL, Morris Q, and Bader GD. 2010. GeneMANIA Cytoscape plugin: fast gene function predictions on the desktop. Bioinformatics 26:2927-2928.

Moschen AR, Kaser A, Enrich B, Mosheimer B, Theurl M, Niederegger H, and Tilg H. 2007. Visfatin, an adipocytokine with proinflammatory and immunomodulating properties. Journal of Immunology 178:1748-1758.

Motawi TM, Shaker OG, Elsawalhi MM, and Abdelnasser ZM. 2014. Visfatin -948G/T and resistin $-420 \mathrm{C} / \mathrm{G}$ polymorphisms in Egyptian type 2 diabetic patients with and without cardiovascular diseases. Genome / National Research Council Canada = Génome / Conseil national de recherches Canada 57:259.

Nguyen MT, Favelyukis S, Nguyen AK, Reichart D, Scott PA, Jenn A, Liu-Bryan R, Glass CK, Neels JG, and Olefsky JM. 2007. A subpopulation of macrophages infiltrates hypertrophic adipose tissue and is activated by free fatty acids via Toll-like receptors 2 and 4 and JNK-dependent pathways. Journal of Biological Chemistry 282:35279-35292.

Ouchi N, Parker JL, Lugus JJ, and Walsh K. 2011. Adipokines in inflammation and metabolic disease. Nature Reviews Immunology 11:85.

Revollo JR, Körner A, Mills KF, Satoh A, Wang T, Garten A, Dasgupta B, Sasaki Y, Wolberger C, and Townsend RR. 2007. Nampt/PBEF/Visfatin regulates insulin secretion in beta cells as a systemic NAD biosynthetic enzyme. Cell Metabolism 6:363-375.

Ritchie ME, Phipson B, Wu D, Hu Y, Law CW, Shi W, and Smyth GK. 2015. limma powers differential expression analyses for RNA-sequencing and microarray studies. Nucleic Acids Research 43:e47.

Saben J, Zhong Y, Gomezacevedo H, Thakali KM, Borengasser SJ, Andres A, and Shankar K. 2013. Early growth response protein-1 mediates lipotoxicity-associated placental inflammation: role in maternal obesity. American Journal of Physiology Endocrinology \& Metabolism 305:E1. 
407

408

409

410

411

412

Schaefergraf UM, Pawliczak J, Passow D, Hartmann R, Rossi R, Bührer C, Harder T, Plagemann A, Vetter K, and Kordonouri O. 2005. Birth weight and parental BMI predict overweight in children from mothers with gestational diabetes. Diabetes Care 28:17451750.

Shen N, Yu X, Pan FY, Gao X, Xue B, and Li CJ. 2011. An Early Response Transcription Factor, Egr-1, Enhances Insulin Resistance in Type 2 Diabetes with Chronic Hyperinsulinism. Journal of Biological Chemistry 286:14508-14515.

Sherlock G. 2009. Gene Ontology: tool for the unification of biology. Canadian Institute of Food Science \& Technology Journal 22:415.

Tack CJ, Stienstra R, Joosten LA, and Netea MG. 2012. Inflammation links excess fat to insulin resistance: the role of the interleukin-1 family. Immunological Reviews 249:239-252.

Thakali KM, Saben J, Faske JB, Lindsey F, Gomezacevedo H, Jr CLL, Badger TM, Andres A, and Shankar K. 2014. Maternal Pre-Gravid Obesity Changes Gene Expression Profiles Towards Greater Inflammation and Reduced Insulin Sensitivity in Umbilical Cord. Pediatric Research 76:202-210.

Tian C, Xing G, P, Lu K, Nie J, Wang J, Li L, Gao M, Zhang L, and He F. 2009. KRAB-type zinc-finger protein Apak specifically regulates p53-dependent apoptosis. Nature Cell Biology 11:580.

Wang D, Guan MP, Zheng ZJ, Li WQ, Lyv FP, Pang RY, and Xue YM. 2015. Transcription Factor Egr1 is Involved in High Glucose-Induced Proliferation and Fibrosis in Rat Glomerular Mesangial Cells. Cellular Physiology \& Biochemistry International Journal of Experimental Cellular Physiology Biochemistry \& Pharmacology 36:2093-2107.

Wang Y, Wang J, Deng Z, Jin J, Jiang H, Meng J, Xu H, Zhao J, Sun G, and Qian H. 2017. Chronic Osteomyelitis Increases the Incidence of Type 2 Diabetes in Humans and Mice. International Journal of Biological Sciences 13:1192-1202.

Whitworth ZK, Mclean SP, and Smith JC. 2017. Social, Environmental, and Racial/Ethnic Factors in Diabetes Prevalence in the U.S.

Wu H, Zhong J, Yu M, Wang H, Gong W, Pan J, Fei F, Wang M, Yang L, and Hu R. 2017. Incidence and time trends of type 2 diabetes mellitus in youth aged 5-19 years: a population-based registry in Zhejiang, China, 2007 to 2013. Bmc Pediatrics 17:85.

Zhao J, Wang C, Xin Y, Zhang S, Yanqin FU, and Endocrinology DO. 2016. Implication of Egr1 on mRNA expressions of PEPCK and G-6-Pase in liver from mice with gestational diabetes mellitus. Journal of Zhengzhou University.

\section{Legends}

Figure 1 Heatmaps of differentially expressed genes

In the right rectangle, red represents a higher expression level, while blue represents a lower expression level. The middle strip indicates the grouping information. The red section is healthy group, while the black section represents the patient group. The right and bottom labels indicate 
445

446

447

448

449

450

451

452

453

454

455

456

457

458

459

460

461

462

463

464

465

466

467

468

469

470

471

grouping information and gene symbols, respectively. The color key is a histogram, located in the upper right corner. The $\mathrm{X}$ axis is a numerical value representing the level of gene expression, and the $\mathrm{Y}$ axis is the number of corresponding squares. This histogram corresponds to the square on the right.

Figure 2 The results of weighted co-expression gene network analysis

An overview of the co-expressed genes in the current study, demonstrating the relevance of gene modules and phenotypes. A. Scale independence used in WGCNA. B. Mean network connectivity of soft-thresholding powers used in WGCNA. A soft threshold of 6 is the most suitable value. C. Cluster dendrogram of the identified co-expression modules. In this figure, each gene is represented as a leaf and corresponds to a color module. Each color indicates that each gene in its corresponding cluster dendrogram belongs to the same module. If some genes have similar changes in expression, then these genes may be functionally related. Moreover, all these genes can further be included into a single module. The grey block represents that the genes that do not co-express with genes of any other color module. D. Module-trait weighted correlations and corresponding P-values for the identified gene module and their clinical status (healthy and children-onset T2D). The label of color on the left represents the strength of correlation, from 1 (red) to -1 (blue).

Figure 3 Protein-protein interaction network

The results of PPI analysis of DEGs in the highly correlated module. Gray lines, purple lines, red lines, and blue lines represent co-expression, co-localization, physical interactions, and shared protein domains, respectively. The link weight is marked with lines of different thicknesses.

Figure 4 The analysis of transcription factor regulatory network

The pink and green nodes represent the important genes identified by previous analysis and the transcription factors that have regulatory relationships, respectively. Some important genes were not pictured in the network because they were single node, which mean no transcription factor linked to them. The different colored arrows indicate the genes regulated by different 
472 transcription factors, making the results easier to observe.

473 Table 1 The characteristics of included cases and controls

$474 \quad$ BMI: Body mass index

475 Table 2 GO analysis of DEGs and genes in highly correlative module

476 GO: gene ontology; DEGs: differentially expressed genes; BP, biological process; CC, 477 cellular component; MF, molecular function; cGMP, cyclic guanosine monophosphate.

478 Table 3 KEGG and Reactome pathway enrichment analyses of DEGs and genes in highly 479 correlative module

480 KEGG: The Kyoto Encyclopedia of Genes and Genomes; DEGs: differentially expressed 481 genes

482 Table 4 Information of critical genes

483 WGCNA: weighted co-expression network analysis; $\operatorname{logFC}$ : $\log 2$ fold change; PPI: protein484 protein interaction network; degree: the number of related genes in a given analysis.

485 Table 5 No. of references of genes in T2D or T1D

486 The number of references related of $\mathrm{T} 2 \mathrm{D}$ or $\mathrm{T} 1 \mathrm{D}$ is given by the comparative 487 toxicogenomics database. T1D: type 1 diabetes; T2D: type 2 diabetes.

488 Code Supplemental File 1 Original code for DEG analysis

489 The program is written in $\mathrm{R}$ and uses the limma algorithm.

490 Code Supplemental File 2 Original code for WGCNA

491 The program is written in R and uses the WGCNA algorithm.

492 Dataset Supplemental File 1 Grouping information for DEG analysis

493 This grouping information is used in the limma algorithm for DEG analysis, including type, 494 group, and gender information for each sample.

495 Dataset Supplemental File 2 Grouping information of WGCNA

496 This grouping information is used for WGCNA, including type, group, and gender 497 information for each sample.

498 Dataset Supplemental File 3 Original results of PPI 
499 The raw results obtained by PPI analysis were exported by the GeneMANIA plugin in 500 Cytoscape.

501 Dataset Supplemental File 4 Results of DEG, WGCNA and PPI analysis of some genes

502 This table contains information about some genes. These genes are present in high 503 correlation modules and differentially expressed genes. Information includes module, $\operatorname{logFC}$, 504 degree in PPI and WGCNA. $\log$ FC: $\log 2$ fold change

505 Dataset Supplemental File 5 Number of references related to T1D for each gene

506 Data on research literature on type 1 diabetes diabetes are downloaded from the 507 Comparative Toxicogenomics Database.

508 Dataset Supplemental File 6 Number of references related to T2D for each gene

509 Data on research literature on T2D downloaded from the Comparative Toxicogenomics 510 Database.

511 Dataset Supplemental File 7 Original results of transcription factor analysis

512 The results were directly derived from Cytoscape's iRegulon plugin, which contains the 513 correspondence between the transcription factor and the target gene and the NES value. 


\section{Table 1 (on next page)}

The characteristics of included cases and controls

BMI: Body mass index 


\begin{tabular}{lcc}
\hline & Children with T2D $(\mathrm{n}=12)$ & Healthy control $(\mathrm{n}=24)$ \\
\hline Age (year, mean $\pm \mathrm{SD})$ & $14.0 \pm 2.3$ & $11.3 \pm 4.6$ \\
Sex $(\%$ female) & 58 & 58 \\
Race & 2 Caucasian & 11 Caucasian \\
& 2 Hispanic & 7 Hispanic \\
& 7 African-American & 6 Mixed or unknown \\
& 1 Asian & ethnicities \\
BMI (mean Z score \pm SD) & $2.33 \pm 0.32$ & Unknown \\
Initial pH less than 7.3 & $17 \%$ & Unknown \\
Initial HbA1c $($ mean $\pm \mathrm{SD})$ & $12.2 \pm 1.5$ & Unknown \\
\hline
\end{tabular}

2

3 Note: BMI: Body mass index 4 


\section{Table 2 (on next page)}

GO analysis of DEGs and genes in highly correlative module

GO: gene ontology; DEGs: differentially expressed genes; BP, biological process; CC, cellular component; MF, molecular function; cGMP, cyclic guanosine monophosphate. 
Table $\amalg$. GO analysis of DEGs and genes in highly correlative module

\begin{tabular}{|c|c|c|c|}
\hline ID & Description & $\begin{array}{l}\text { No. of } \\
\text { genes }\end{array}$ & P-value \\
\hline Upregulated genes & $\square$ & & \\
\hline GO-BP terms & $\square$ & & \\
\hline GO:0042107 & cytokine metabolic process & 6 & $3.02 \mathrm{E}-04$ \\
\hline GO:0042089 & cytokine biosynthetic process & 6 & $2.76 \mathrm{E}-04$ \\
\hline GO:0032611 & $\begin{array}{l}\text { interleukin- } 1 \text { beta production } \\
\text { positive regulation of cytokine }\end{array}$ & 5 & $2.53 \mathrm{E}-04$ \\
\hline GO:0042108 & $\begin{array}{l}\text { biosynthetic process } \\
\text { MyD88-dependent toll-like receptor }\end{array}$ & 5 & $1.47 \mathrm{E}-04$ \\
\hline GO:0002755 & $\begin{array}{l}\text { signaling pathway } \\
\text { positive regulation of interleukin-1 }\end{array}$ & 5 & 8.59E-06 \\
\hline GO:0032732 & production & 4 & 4.47E-04 \\
\hline GO:0045351 & $\begin{array}{l}\text { type I interferon biosynthetic process } \\
\text { positive regulation of interferon-beta }\end{array}$ & 4 & $2.19 \mathrm{E}-06$ \\
\hline GO:0032728 & $\begin{array}{l}\text { production } \\
\text { positive regulation of interleukin-1 }\end{array}$ & 4 & $9.85 \mathrm{E}-05$ \\
\hline GO:0032731 & beta production & 4 & 2.39E-04 \\
\hline GO:0050702 & interleukin-1 beta secretion & 4 & 4.85E-04 \\
\hline GO:0006491 & N-glycan processing & 3 & $3.84 \mathrm{E}-04$ \\
\hline GO:0034755 & iron ion transmembrane transport & 3 & 4.49E-04 \\
\hline GO:0042228 & $\begin{array}{l}\text { interleukin- } 8 \text { biosynthetic process } \\
\text { regulation of interleukin- } 8\end{array}$ & 3 & $1.84 \mathrm{E}-04$ \\
\hline GO:0045414 & biosynthetic process & 3 & $1.48 \mathrm{E}-04$ \\
\hline GO:0045350 & interferon-beta biosynthetic process & 3 & $3.52 \mathrm{E}-05$ \\
\hline GO:0045357 & regulation of interferon-beta & 3 & $3.52 \mathrm{E}-05$ \\
\hline
\end{tabular}


GO:0045416

GO:0045359

GO:0045356

Downregulated genes

GO-BP terms

GO:0001945

GO:0060613

Blue-Cyan module

GO-BP terms

GO:0070647

GO:0032446

GO:0006396

GO:0006397

GO:0008380

GO:0007034

GO:0007041

GO:0007029

GO:0071786

GO-CC terms

GO:0043233 biosynthetic process

positive regulation of interleukin-8

biosynthetic process

$3 \quad 5.00 \mathrm{E}-05$

positive regulation of interferon-beta

biosynthetic process

$3 \quad 1.48 \mathrm{E}-05$

positive regulation of interferon-

alpha biosynthetic process

2

$3.43 \mathrm{E}-04$

$4 \quad 1.81 \mathrm{E}-04$

$3 \quad 2.62 \mathrm{E}-05$

fat pad development

protein modification by small protein conjugation or removal

$59.00 \quad 2.74 \mathrm{E}-05$

protein modification by small protein

conjugation

$51.00 \quad 2.49 \mathrm{E}-05$

RNA processing

$51.00 \quad 5.13 \mathrm{E}-05$

mRNA processing

35.00

$2.44 \mathrm{E}-06$

RNA splicing

$29.00 \quad 7.10 \mathrm{E}-05$

vacuolar transport

$13.00 \quad 5.86 \mathrm{E}-05$

lysosomal transport

$11.00 \quad 1.04 \mathrm{E}-04$

endoplasmic reticulum organization

$8.00 \quad 3.32 \mathrm{E}-05$

endoplasmic reticulum tubular

$5.00 \quad 9.27 \mathrm{E}-05$

network organization

organelle lumen

1.49E-09 


$\begin{array}{llrr}\text { GO:0070013 } & \text { intracellular organelle lumen } & 216.00 & 1.49 \mathrm{E}-09 \\ \text { GO:0044428 } & \text { nuclear part } & 203.00 & 4.75 \mathrm{E}-13 \\ \text { GO:0031981 } & \text { nuclear lumen } & 189.00 & 1.36 \mathrm{E}-12 \\ \text { GO:0005654 } & \text { nucleoplasm } & 164.00 & 1.55 \mathrm{E}-11 \\ \text { GO:0044451 } & \text { nucleoplasm part } & 60.00 & 5.83 \mathrm{E}-06 \\ \text { GO-MF terms } & \square & \square & \square \\ \text { GO:0003723 } & \text { RNA binding } & 79.00 & 1.65 \mathrm{E}-05 \\ & \text { ubiquitin-like protein transferase } & 31.00 & 2.04 \mathrm{E}-05 \\ \text { GO:0019787 } & \text { activity } & & \\ \text { GO:0004842 } & \text { ubiquitin-protein transferase activity } & 30.00 & 2.01 \mathrm{E}-05\end{array}$

1 GO: gene ontology; DEGs: differentially expressed genes; BP, biological process; CC, cellular 2 component; MF, molecular function; cGMP, cyclic guanosine monophosphate. 


\section{Table 3 (on next page)}

KEGG and Reactome pathway enrichment analyses of DEGs and genes in highly correlative module

KEGG: The Kyoto Encyclopedia of Genes and Genomes; DEGs: differentially expressed genes 
Table III. KEGG and Reactome pathway enrichment analyses of DEGs and genes in highly correlative module

\begin{tabular}{lcc}
\hline Description & No. of & P-value \\
& genes & \\
\hline
\end{tabular}

Upregulated genes

KEGG

Toll-like receptor signaling pathway

5

$1.16 \mathrm{E}-03$

Reactome Pathways

R-HSA:168898

Toll-Like Receptors Cascades

6

1.31E-03

RAB GEFs exchange GTP for GDP

R-HSA:8876198

on RABs

5

$6.00 \mathrm{E}-04$

R-HSA:5260271

Diseases of Immune System

3

1.09E-03

Diseases associated with the TLR

R-HSA:5602358

signaling cascade

3

1.09E-03

Downregulated genes

Reactome Pathways

R-HSA:3247509

Chromatin modifying enzymes

10

3.10E-04

R-HSA:4839726

Chromatin organization

10

3.10E-04

R-HSA:72163

mRNA Splicing - Major Pathway

8

3.76E-04

R-HSA:72172

mRNA Splicing

8

5.00E-04

Blue-Cyan module

KEGG

Vasopressin-regulated water

KEGG:04962

Reabsorption

3

1.12E-03

Reactome Pathways

R-HSA:909733 Interferon alpha/beta signaling

3

4.08E-03

R-HSA:8852135 Protein ubiquitination

5.55E-03 


$\begin{array}{llll} & \text { Synthesis of active ubiquitin: roles of } & \\ \text { R-HSA:8866652 } & \text { E1 and E2 enzymes } & 3 & 3.60 \mathrm{E}-04 \\ \text { R-HSA:983152 } & \text { Transfer of ubiquitin from E1 to E2 } & 3 & 1.05 \mathrm{E}-03 \\ \text { R-HSA:193648 } & \text { NRAGE signals death through JNK } & 2 & 3.10 \mathrm{E}-02 \\ \text { R-HSA:933528 } & \text { Interaction of MEKK1 with TRAF6 } & 2 & 5.91 \mathrm{E}-04 \\ \text { R-HSA:933530 } & \text { Activation of IKK by MEKK1 } & 2 & 1.15 \mathrm{E}-03\end{array}$

1 KEGG: The Kyoto Encyclopedia of Genes and Genomes; DEGs: differentially expressed genes 


\section{Table 4 (on next page)}

Information of critical genes

WGCNA: weighted co-expression network analysis; logFC: log2fold change; PPI: proteinprotein interaction network; degree: the number of related genes in a given analysis. 
Table IV. Information of critical genes

\begin{tabular}{ccccc}
\hline Gene Symbol & WGCNA module & $\operatorname{logFC}$ & WGCNA degree(n) & PPI \\
\hline TRAPPC11 & blue & 1.292140612 & 193 & 8 \\
CHM & blue & 0.801222752 & 138 & 12 \\
C14orf119 & blue & 0.777600335 & 262 & 16 \\
ETNK1 & blue & 0.744750579 & 161 & 11 \\
GSKIP & blue & 0.697264964 & 317 & 11 \\
PIK3CG & blue & 0.688131993 & 178 & 13 \\
ZNF420 & blue & 0.656561519 & 96 & 7 \\
QSER1 & blue & 0.653280142 & 126 & 10 \\
RAB10 & blue & 0.639129192 & 115 & 12 \\
MRPL35 & blue & 0.638260852 & 240 & 7 \\
\hline
\end{tabular}

WGCNA: weighted co-expression network analysis; $\log _{\mathrm{FC}}$ : $\log _{2}$ fold change; PPI: proteinprotein interaction network; degree: the number of related genes in a given analysis. 


\section{Table 5 (on next page)}

No. of references of genes in T2D or T1D

The number of references related of T2D or T1D is given by the comparative toxicogenomics database. T1D: type 1 diabetes; T2D: type 2 diabetes. 
Table V. No. of references of genes in T2D or T1D

\begin{tabular}{ccc}
\hline Gene & T2D(n) & T1D(n) \\
\hline EGR1 & 104 & 14 \\
NAMPT & 99 & 26 \\
MRPL35 & 27 & 2 \\
QSER1 & 25 & 1 \\
ETNK1 & 11 & 2 \\
RAB10 & 9 & 5 \\
CHM & 6 & 2 \\
PIK3CG & 6 & 6 \\
TRAPPC11 & 4 & 2 \\
C14orf119 & 4 & 1 \\
GSKIP & 1 & 2 \\
ZNF420 & 1 & 1 \\
\hline
\end{tabular}

1 The number of references related of T2D or T1D is given by The comparative toxicogenomics

2 database. T1D: type 1 diabetes; T2D: type 2 diabetes. 


\section{Figure 1}

Heatmaps of differentially expressed genes

In the right rectangle, red represents a higher expression level, while blue represents a lower expression level. The middle strip indicates the grouping information. The red section is healthy group, while the black section represents the patient group. The right and bottom labels indicate grouping information and gene symbols, respectively. The color key is a histogram, located in the upper right corner. The $\mathrm{X}$ axis is a numerical value representing the level of gene expression, and the $\mathrm{Y}$ axis is the number of corresponding squares. This histogram corresponds to the square on the right.

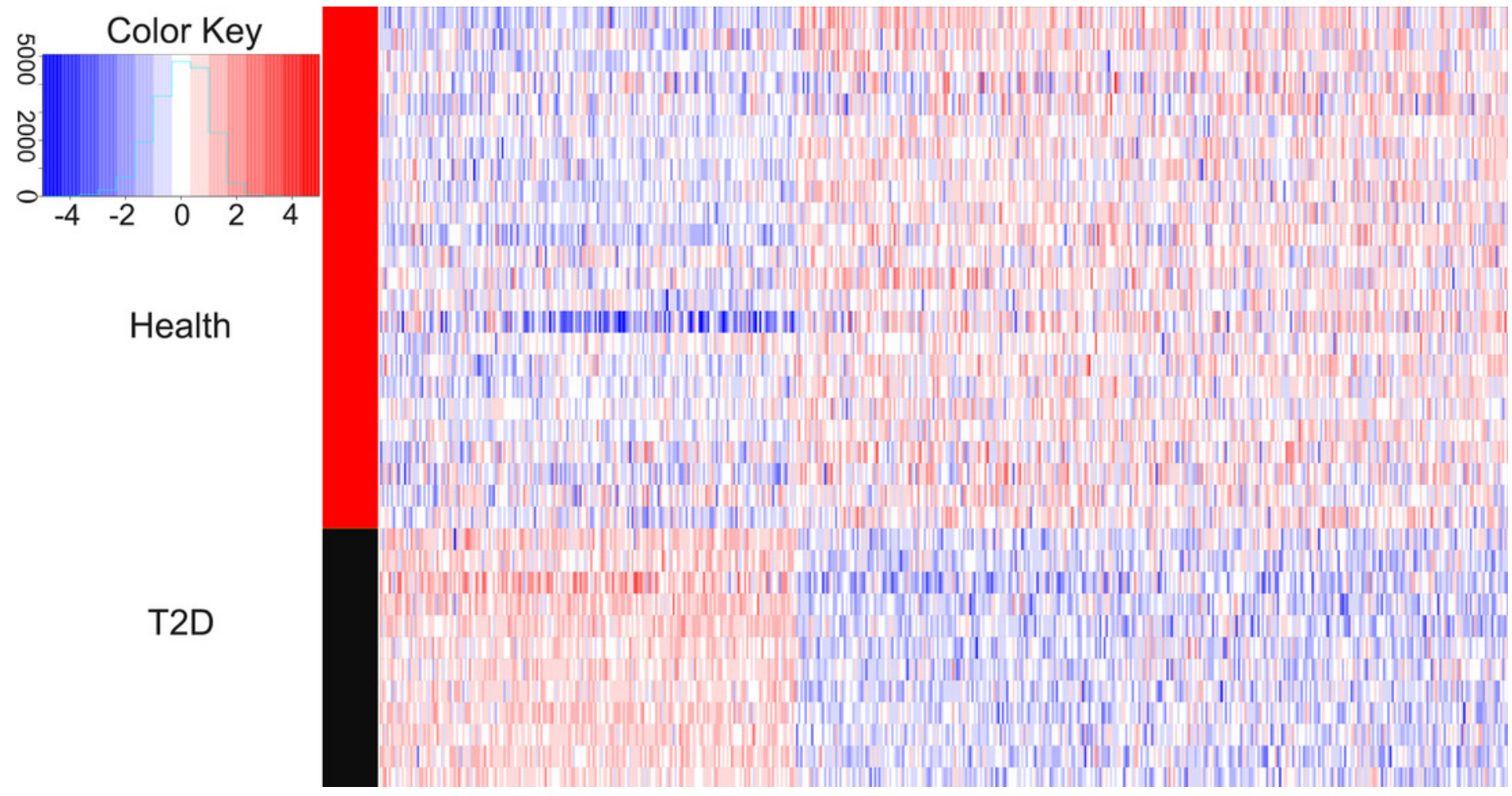




\section{Figure 2}

The results of weighted co-expression gene network analysis

An overview of the co-expressed genes in the current study, demonstrating the relevance of gene modules and phenotypes. A. Scale independence used in WGCNA. B. Mean network connectivity of soft-thresholding powers used in WGCNA. A soft threshold of 6 is the most suitable value. C. Cluster dendrogram of the identified co-expression modules. In this figure, each gene is represented as a leaf and corresponds to a color module. Each color indicates that each gene in its corresponding cluster dendrogram belongs to the same module. If some genes have similar changes in expression, then these genes may be functionally related. Moreover, all these genes can further be included into a single module. The grey block represents that the genes that do not co-express with genes of any other color module. D. Module-trait weighted correlations and corresponding P-values for the identified gene module and their clinical status (healthy and children-onset T2D). The label of color on the left represents the strength of correlation, from 1 (red) to -1 (blue). 
A Scale independence

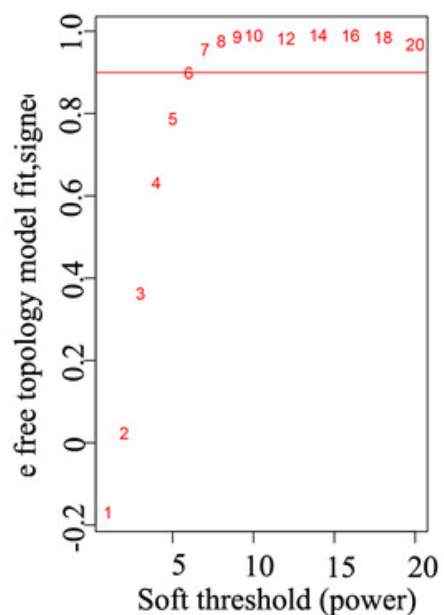

B

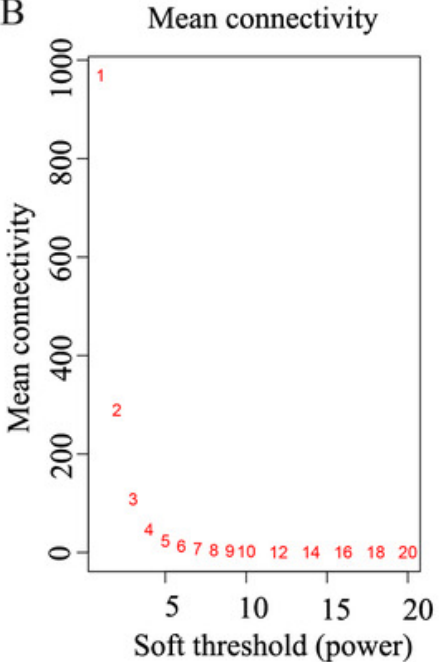

$\mathrm{C}$

Cluster dendrogream

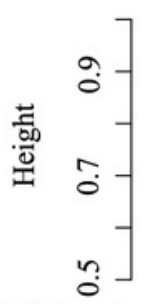

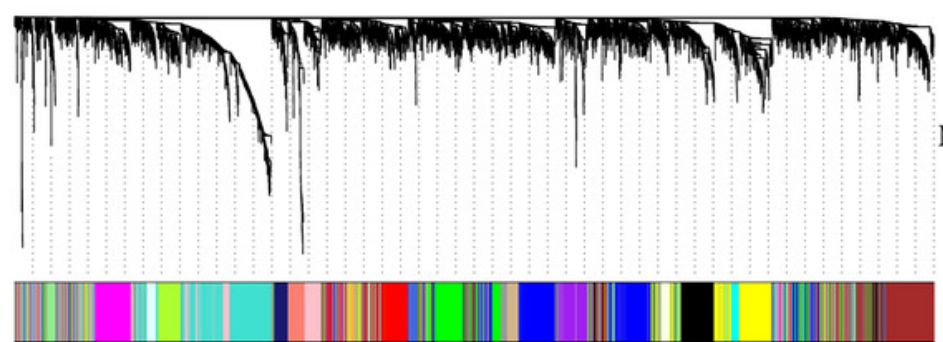

D

\section{Module-trait relationships}

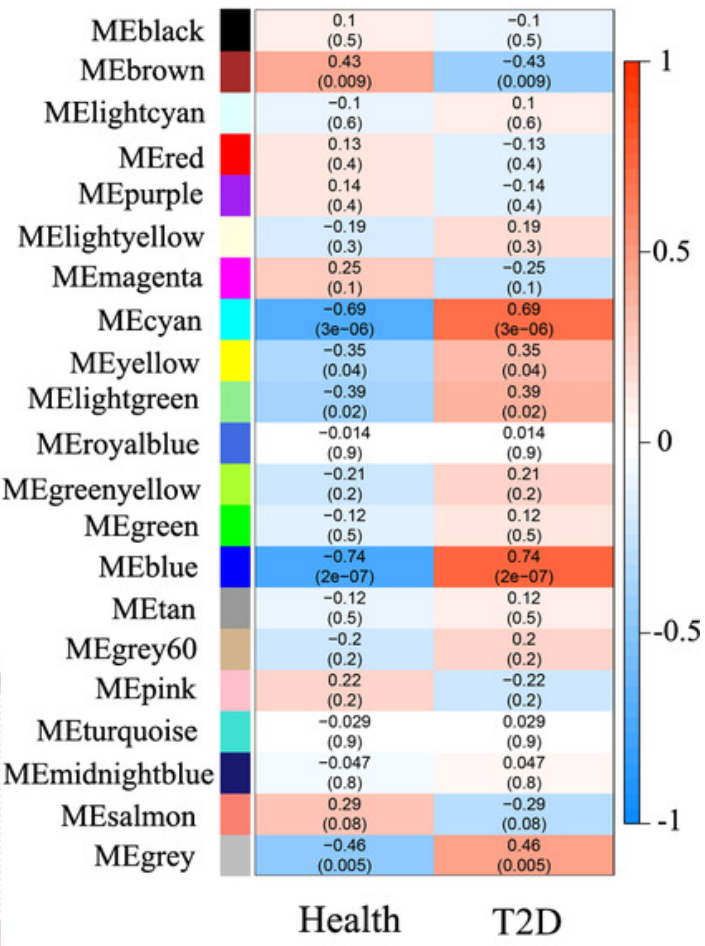




\section{Figure 3}

\section{Protein-protein interaction network}

The results of PPI analysis of DEGs in the highly correlated module. Gray lines, purple lines, red lines, and blue lines represent co-expression, co-localization, physical interactions, and shared protein domains, respectively. The link weight is marked with lines of different thicknesses.

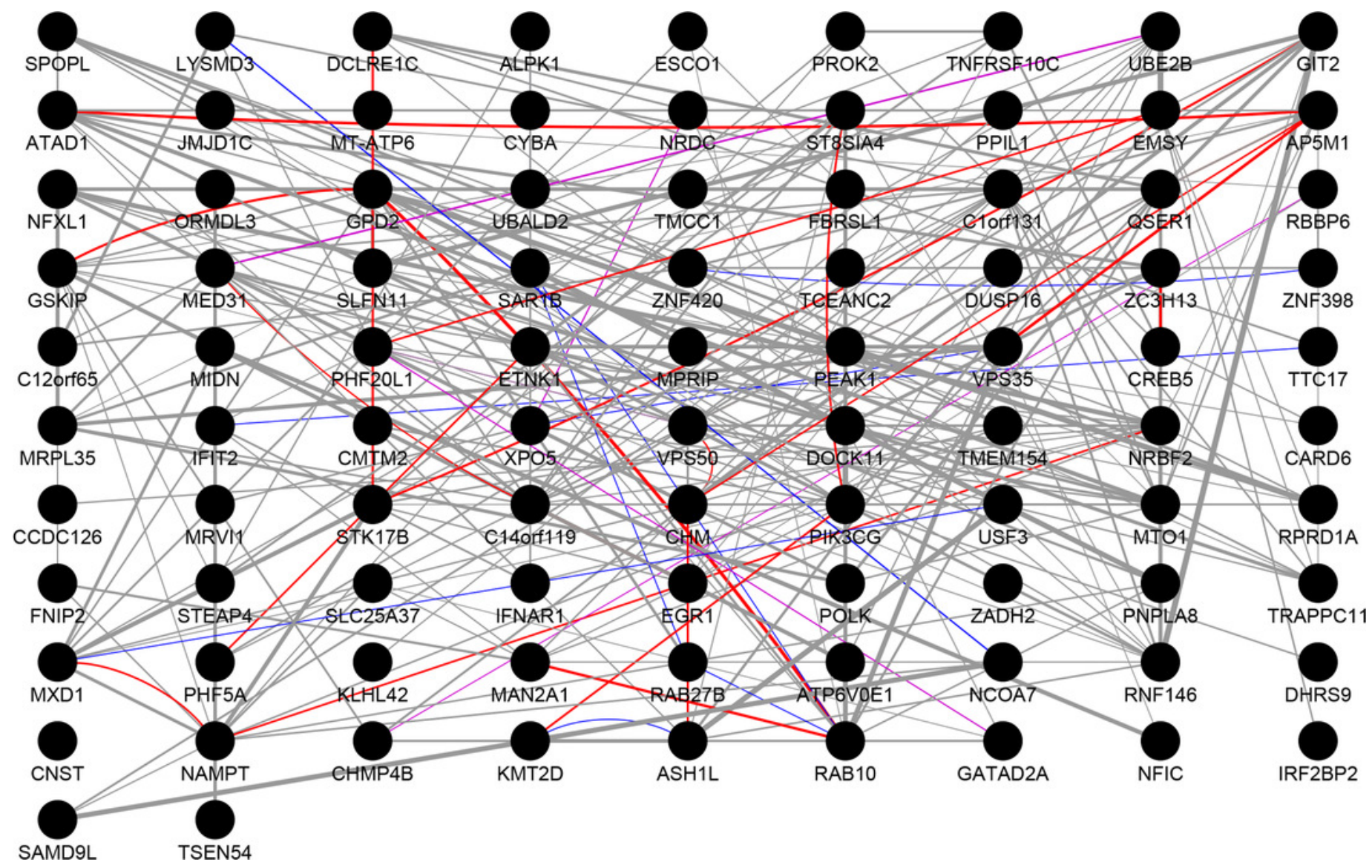




\section{Figure 4}

The analysis of transcription factor regulatory network

The pink and green nodes represent the important genes identified by previous analysis and the transcription factors that have regulatory relationships, respectively. Some important genes were not pictured in the network because they were single node, which mean no transcription factor linked to them. The different colored arrows indicate the genes regulated by different transcription factors, making the results easier to observe. 


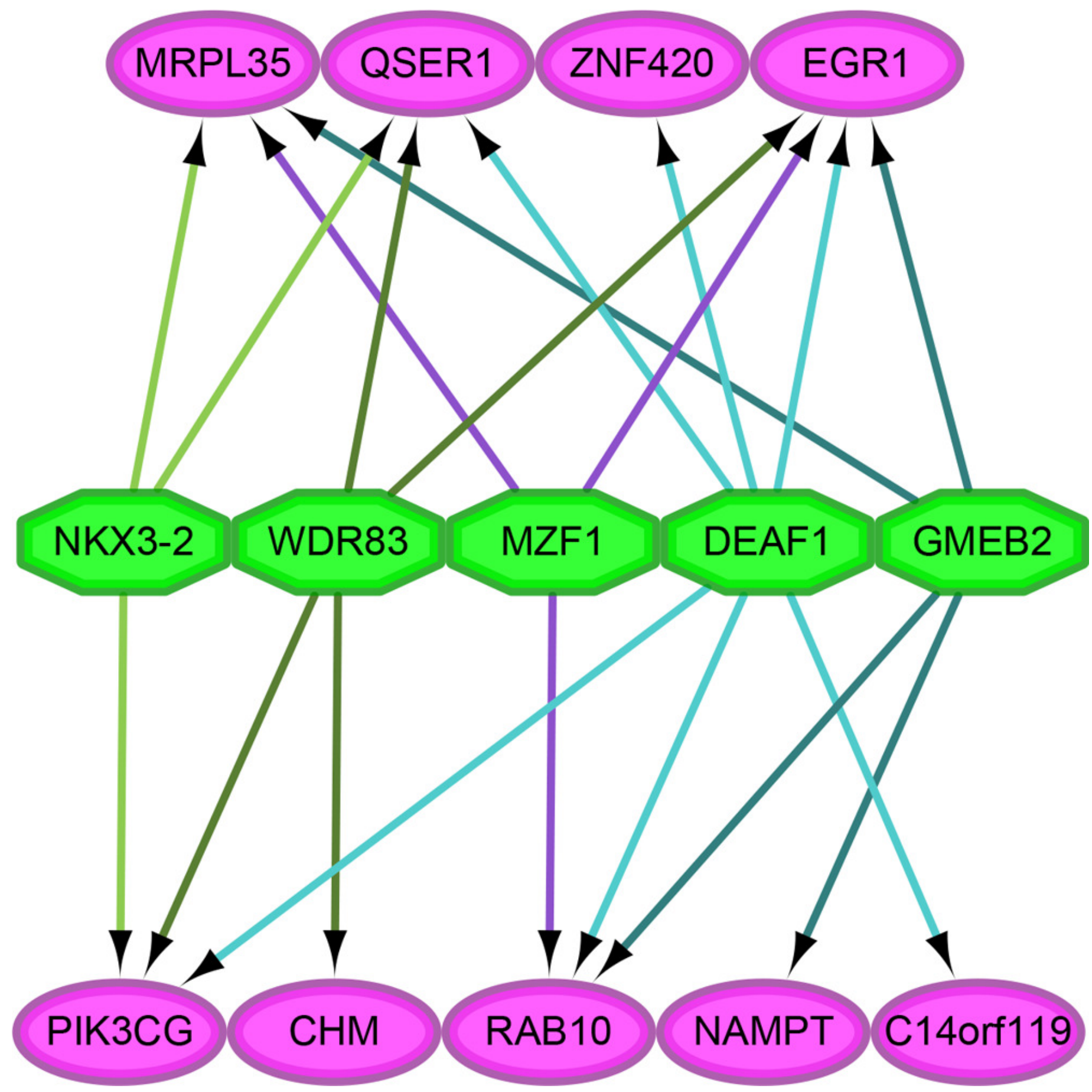

